\title{
VLF Detection of Fireballs
}

by

Martin Beech ${ }^{1}$, Peter Brown ${ }^{2}$, and J. Jones ${ }^{2}$.

1 Department of Astronomy, 2 Department of Physics, University of Western Ontario, London, Ontario, Canada, N6A 3K7.

\begin{abstract}
The results from 80 hours of simultaneous visual/video and VLF recordings made during the Lyrids, Perseids, Orionids, Leonids and Geminids are presented. All meteor magnitudes from -11 to +4 have been sampled at least once during these observations. The only positive VLF fireball detection was made at 19:57:32 UT on August 11, 1993 from the South of France. We present a Fourier transform analysis of this event and we also derive a lower limit to the electrical field strength produced by the fireball at the antenna. Our present observations suggest a lower limit of $M_{V} \approx-10 \pm 1$ for a fireball to produce a VLF signal.
\end{abstract}




\section{Introduction}

The possibility that bright meteors might generate intrinsic radio emissions was first mooted some sixty years ago. Bunch (1930), for example, suggested that "meteor static" might be "made audible with a radio receiving set." Bunch also noted that the anomalous (now electrophonic) sounds heard during the passage of bright fireballs might also have an "electrical" origin. The first detailed investigations into the possibility that meteors might generate radio emissions were those of Hawkins (1958a, 1958b). Hawkins found no evidence to support the radio emission hypothesis, and, in fact, commented that meteors showed a "surprisingly low efficiency in converting kinetic to radio energy."

More recently, it has been proposed that the electrophonic sounds which occasionally accompany bright fireballs are caused by the transduction into sound of meteor generated ELF/VLF radiation (Bronshtén, 1983, and Keay $1980,1992,1994)$. Several observational accounts have been published suggesting the positive detection of meteor generated VLF radiation. Watanabe et al. (1988) have described the detection of VLF emission from a bright Perseid fireball $\left(M_{V} \approx-6\right)$ observed in 1981. Drobnock (1992) has also suggested that meteors as faint as $\mathrm{M}_{\mathrm{V}} \approx+1$ can generate VLF emissions.

\section{Observations - phase 1:}

The present study was motivated by the extraordinary claim by Drobnock (1992) that 'ordinary' meteors can produce VLF emissions. Our early studies, using simply transistor circuits, failed to indicate any obvious correlations between the appearance of 'typical' meteors and the generation of a distinct VLF signal. Continued observations with a much more sensitive system (see figure 1) have also failed to verify Drobnock's observations. A record of our observing sessions is given in table 1.

It is our belief that Drobnock has confused natural VLF radio background emissions for coincident signals. Indeed it is important to realize that VLF radio emissions are continually generated in the Earth's ionosphere. These 


\section{VLF Meteor Observing Log}

\begin{tabular}{lccccc}
\hline Shower & Year & $\begin{array}{l}\mathrm{V}_{\infty} \\
\left(\mathrm{kms}^{-1}\right)\end{array}$ & $\mathrm{r}$ & $\begin{array}{l}\mathrm{H}_{\text {obs. }} \\
(\mathrm{hr})\end{array}$ & $\begin{array}{l}\text { Max. } \\
\mathrm{M}_{\mathrm{v}}\end{array}$ \\
\hline Lyrids & 1993 & 49 & 2.9 & 6.0 & -1.5 \\
& 1994 & - & - & 12.5 & -3 \\
Perseids & 1992 & 59 & 2.6 & 10.0 & -6 \\
& 1993 & - & - & 28.0 & -11 \\
& 1994 & - & - & 10.0 & -8 \\
Orionids & 1992 & 66 & 2.9 & 2.0 & +0.5 \\
Leonids & 1993 & 71 & 2.5 & 3.0 & +1 \\
Geminids & 1993 & 35 & 2.6 & 8.75 & -8 \\
\hline Sporadic & - & - & - & 80.25 & -10 \\
\hline
\end{tabular}

Table 1. Column one indicates the various meteor showers that we have monitored. Column two shows the year in which the observations were made. Columns three and four show the typical velocity $\left(\mathrm{V}_{\infty}\right)$ and population index (r) for each stream observed. Column five gives the number of hours that the stream was monitored, and column six shows the visual magnitude of the brightest meteor observed in each monitoring session.

Table 1. VLF meteor observing log. Column one identifies the various meteor showers that we have monitored. Column two shows the year in which the observations were made. Columns three and four show the typical velocity $\left(\mathrm{V}_{\infty}\right)$ and the population index $(\mathrm{r})$ for each stream observed. Column five gives the number of hours that the stream was monitored, and column six shows the visual magnitude of the brightest meteor observed in each session. 


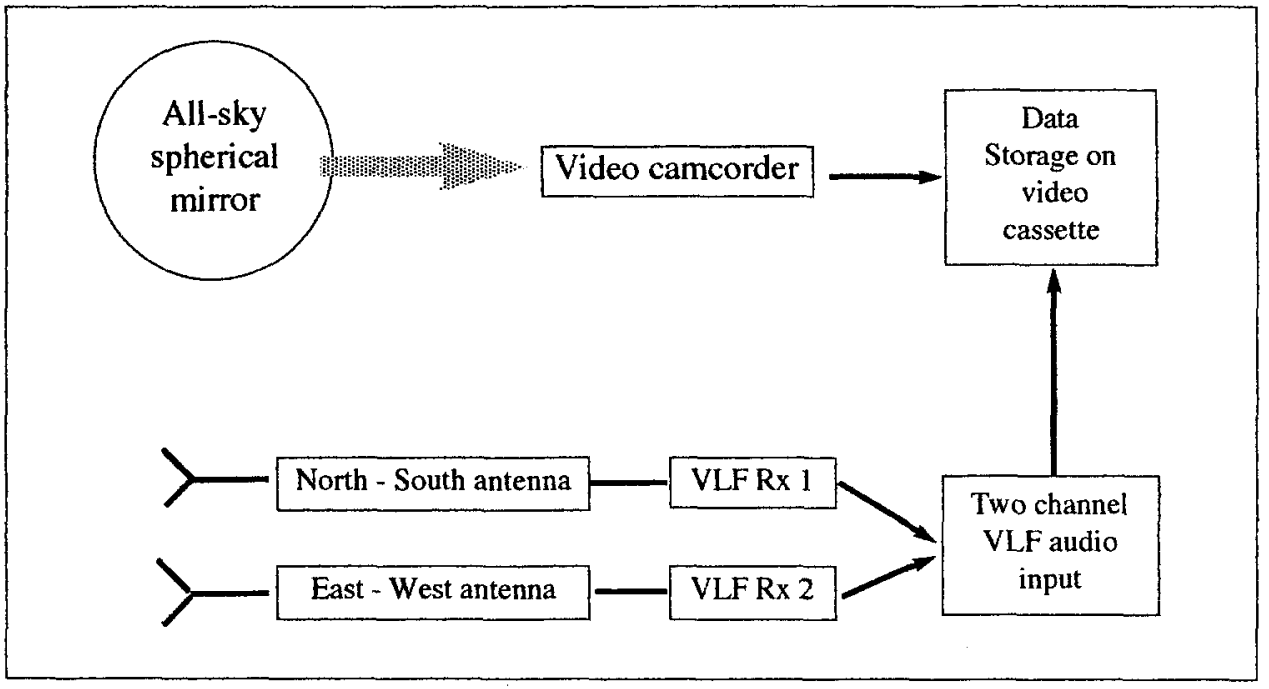

Figure 1. Schematic diagram of the coincidence detector used in Puimichel, France. The video recorder employed was a Samsung SCX 953 video camcorder. The VLF receivers are identical, variable gain $\left(\leq 10^{5}\right)$ amplifiers with built in high and low pass filters. The low end cut-off for each receiver is $1 \mathrm{kHz}$. The antenna loop area is $1 \mathrm{~m}^{2}$.

emissions show a wide variety of tonal characteristics (Okada and Iwai, 1988). We commonly record pure tone sources, rising and falling tone sources (whistlers) and discrete emissions which produce various 'clicks' and rapid tone changes. Many every-day objects also produce VLF emissions. In order to reduce interference from electrical transmission cables, passing cars, and domestic sources we have introduced both high and low pass filters to our system. Care does have to be exercised in the identification of coincidences between VLF emission changes and the appearance of meteors.

\section{Observations - phase 2:}

In addition to testing the claim that 'ordinary' meteors might generate VLF radio signals, we also wanted to investigate the possibility that the less common bright fireballs can produce VLF radiation. Keay (1980, 1992, 1994), for example, has outlined several mechanisms by which bright fireballs can produce VLF emissions. Two mechanisms in particularly 
seem worthy of further study. In the first mechanism it is the trapping and twisting of the Earth's magnetic field in the fireball's turbulent wake that produces VLF radiation. While in the second mechanism VLF radiation is produced by the rapid expulsion, and subsequent relaxation of the Earth's magnetic field from a region surrounding the fireball.

The magnitude distribution of bright $\left(\mathrm{M}_{\mathrm{V}} \leq 0\right)$ meteors observed during the past three years is given in table 2 . We have now sampled many fireball events, the brightest of which had a maximum visual magnitude of $-11 \pm 1$ mag. Only one of the fireball events recorded has produced a clear, and convincing VLF signal.

\begin{tabular}{|llllllllllllll|}
\hline \multicolumn{11}{|c|}{ Observed Magnitude Distribution: $M_{V} \geq 0$} \\
\hline$M_{V}$ & 0 & -1 & -2 & -3 & -4 & -5 & -6 & -7 & -8 & -9 & -10 & -11 & -12 \\
\hline No. & 28 & 24 & 9 & 12 & 10 & 9 & 3 & 2 & 3 & 1 & 1 & 1 & 0 \\
\hline
\end{tabular}

Table 2. Magnitude distribution of the brightest meteors observed. A complete count of meteors fainter than $M_{V} \approx 0$ has not been kept.

At 19:57:32 UT on August 11, 1993 a bright, visual magnitude $-10 \pm 1$ fireball was observed from Puimichel, in Southern France. Concomitant to the appearance of the fireball a unique (to our observing experience) VLF signal was recorded. The optical 'flash' produced by the fireball's terminal flare, and the simultaneous 'buzz' from the audio output of the VLF receivers was noted by one of the authors $(\mathrm{MB})$ and two other observers.

The fireball appeared in the north-eastern part of the sky, and was reasonably well placed for our antenna gain pattern. The fireball was, however, below the detection horizon of our all-sky mirror.

The time-resolved signal strength variations, and its associated Fourier transform are shown in figure 2 . The rapid $(\approx 6 \mathrm{kHz})$ signal modulation is due to the recording characteristics of the camcorder. Points $\mathrm{B}$ and $\mathrm{E}$ indicate the beginning and end of the signal. At point $G$ the camcorder's 


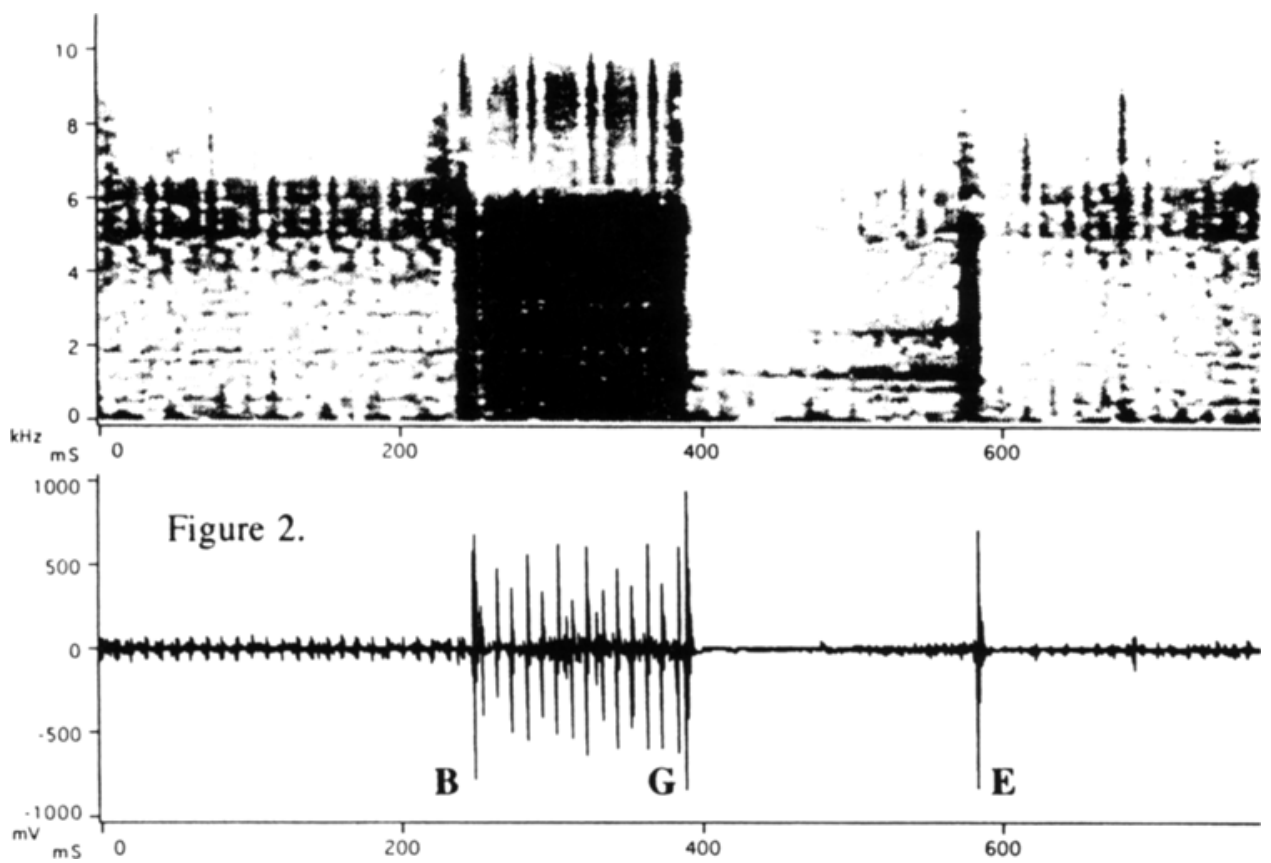

Figure 2. Characteristics of the August 11, 1993 fireball event. Upper panel: Fourier transform of the recorded signal variations. Lower panel: Signal strength variations. Point $B$ and $E$ indicate the beginning and end of the event. At point $G$ the camcorder's automatic gain control was activated.

automatic gain control was activated. The time interval $\mathrm{B}$ to $\mathrm{E}$ is about $1 / 3$ rd of a second long. The Fourier transform analysis indicates that the signal has a broad band component between 1 and $10 \mathrm{kHz}$, with two reasonably well established peaks near 1.5 and $2.5 \mathrm{kHz}$.

\section{Energetics of the 1993 Event}

From the known system characteristics and a few simplifying assumptions it is possible to estimate the local electrical field strength, Eo, generated by the fireball's passage. An application of Faraday's law indicates that the magnetic field strength at the antenna, Bo, must be of order

Bo $>1$ volt $/\left(2 \times 10^{3} \mathrm{~Hz}\right)(360)\left(1 \mathrm{~m}^{2}\right) \approx 8 \times 10^{-6}$ Tesla 
Where the 1 volt term corresponds to the induced emf. The emf must have been at least this value since the automatic gain control is triggered at 1 volt. A characteristic magnetic field strength variation of $2 \mathrm{kHz}$ has been adopted upon the basis of the Fourier analysis (see figure 2). The antenna has an area of $1 \mathrm{~m}^{2}$, and contains 360 turns of wire. The electrical field strength at the antenna will be $E o=c B o$, where $c$ is the speed of light. We find that Eo $\geq 2 \times 10^{3}$ volt $/ \mathrm{m}$.

No accounts of electrophonic sounds, or sonic booms were reported for the August 11th, 1993 event. This, however, is not too surprising given the short duration of the event, and the fact that it occurred early-on in the evening before most observers were active.

\section{Discussion}

The observations that we have carried out to date have been made in the 1 to $10 \mathrm{kHz}$ region. We believe that our detection system is of a sufficient sensitivity to claim, with table 2 as a guide, that most fireballs do not produce VLF emissions. Our observations suggest that fireballs fainter than $M_{V} \approx-10 \pm 1$ are not likely to generate VLF emissions. Our observations also indicate that neither meteoroid velocity (see table 1), train duration or the presence of a terminal flare appear to influence the likelihood that fireball's fainter than $M_{V} \approx-8$ will produce VLF emissions. We find absolutely no evidence to support the claim made by Drobnock (1992) that meteors as faint as $M_{V} \approx+1$ can produce VLF radio emissions.

\section{References}

Bronshtén, V. A. 1983. In Physics of Meteoric Phenomena. D.Reidel, Dordrecht.

Bunch, S. 1930. Popular Astronomy, 38, 448.

Drobnock, G. J. 1992. Sky and Telescope, March, 329.

Hawkins, G. S. 1958a. Nature, 181, 1610.

Hawkins, G. S. 1958b. Ap. J. 128, 724.

Keay, C. S. L. 1980. Science, $210,11$.

Keay, C. S. L. 1992. Meteoritics, 27, 144. 
Keay, C. S. L. 1994. Journal of Scientific Exploration, 7, 337.

Okada, T., and Iwai, A. 1988. Natural VLF Radio Waves. Research Studies Press Ltd, Letchworth, Hertfordshire.

Watanabe, T., Okada, T., and Suzuki, K. 1988. Ham Journal., \#54, 109.

Acknowledgments: We are grateful to Professor Paul Handford of the Zoology Department at the UWO for his help in generating the voltage amplitude and FFT images. 